\title{
CLIFFORD ALGEBRA APPROACH TO GRAPHENE FLATLAND
}

\author{
A. Dargys \\ Semiconductor Physics Institute, Center for Physical Sciences ant Technology A. Goštauto 11, LT-01108 Vilnius, Lithuania \\ E-mail: dargys@pfi.lt
}

Received 18 November 2013; accepted 4 December 2013

\begin{abstract}
Quantum properties of a monolayer graphene are studied in terms of the Clifford algebras. The simplest of algebras suitable for this purpose appears to be $\mathrm{Cl}_{30}$ algebra, the basis vectors of which represent 3D rather than 2D Euclidean space. If electron spin is included, higher dimensional algebras should be used. It is shown that $\mathrm{Cl}_{3,1}$ algebra which describes Minkowski's spacetime is suitable for this purpose. It is shown that in both algebras, $\mathrm{Cl}_{3,0}$ and $\mathrm{Cl}_{3,1}$ the conduction and valence band spinors are rotors of 3D Euclidean space. Properties of electron spin and Berry phase when exchange and spin-orbit interactions are taken into account are illustrated within Clifford algebra formalism.
\end{abstract}

Keywords: graphene, Clifford algebra, theory and modelling

PACS: $81.05 . \mathrm{Uw}$, 03.65.Pm, 73.43.Cd

\section{Introduction}

The standard quantum mechanics is formulated in the Hilbert space, where quantum states of objects are described by complex wave functions or spinors. The transitions between the states are defined by appropriate operators (complex matrices). However, a different approach to quantum mechanics is possible which is not connected with the Hilbert space. The new approach relies on noncommutative Clifford algebra $\mathrm{Cl}_{p, q}$, also called geometric algebra (GA), where the indices $p$ and $q$ represent the metric of space [1]]. For example, $\mathrm{Cl}_{3,0}$ describes Euclidean 3D space, while $\mathrm{Cl}_{3,1}$ and $\mathrm{Cl}_{1,3}$ describe Minkowski's spacetime. Therefore, selection of a particular GA automatically defines the geometry of space. This allows one to solve the quantum mechanical, electrodynamics and gravitational problems in a more efficient coordinate-free way [1-3]. In this report we consider the prospects of application of the Clifford algebra to graphene which, as known, incorporates a number of relativistic properties, such as Dirac spectrum and Klein tunnelling. In particular, we shall consider the Hamiltonian of a monolayer graphene when electron spin is neglected and included.

\section{Spinless graphene in terms of $\mathrm{Cl}_{3,0}$ algebra}

$\mathrm{Cl}_{3,0}$ algebra consists of scalar, three vectors $\sigma_{1}, \sigma_{2}, \sigma_{3}$, three bivectors $I \sigma_{1}, I \sigma_{2}, I \sigma_{3}$, and pseudoscalar $I=\sigma_{1} \sigma_{2} \sigma_{3}$. The time-dependent Schrodinger-Pauli equation for a monolayer graphene in terms of $\mathrm{Cl}_{3,0}$ reads [4]:

$$
\partial_{t} \psi I \sigma_{3}=\mathbf{k} \psi \sigma_{3} \text {. }
$$

Here $\mathbf{k}=k_{x} \boldsymbol{\sigma}_{1}+k_{y} \boldsymbol{\sigma}_{2}$ is the electron wave vector and $\psi=a_{0}+a_{1} I \sigma_{1}+a_{2}^{y} I \sigma_{2}+a_{3} I \sigma_{3}$ is the spinor, where $a_{i} \mathrm{~s}$ are the real numbers. Note that $\boldsymbol{\sigma}_{i} \sigma_{j}+\sigma_{j} \sigma_{i}=2 \delta_{i j}$. The role of the imaginary unit here is played by the bivector $I \sigma_{3}=\sigma_{1} \sigma_{2}$.

The right-hand side of $(1), H_{30}(\psi)=\mathbf{k} \psi \boldsymbol{\sigma}_{3}$, represents the Hamiltonian function which can be rewritten as a sum of two projectors $P_{+}(\psi)$ and $P_{-}(\psi)$ in the following way:

$$
\begin{aligned}
H_{30}(\psi) & =k\left[\frac{1}{2}\left(\psi+\hat{\mathbf{k}} \psi \boldsymbol{\sigma}_{3}\right)-\frac{1}{2}\left(\psi-\hat{k} \psi \boldsymbol{\sigma}_{3}\right)\right] \\
& =k h_{+} P_{+}(\psi)+k h_{-} P_{-}(\psi),
\end{aligned}
$$

where $k=|\mathbf{k}|=\sqrt{k_{x}^{2}+k_{y}^{2}}, \hat{\mathrm{k}}=\mathbf{k} / k$ is the unit wave vector, and $h_{ \pm}= \pm 1$ represents the helicity quantum number. The projectors $P_{ \pm}(\psi)=\frac{1}{2}\left(\psi \pm \hat{\mathbf{k}} \psi \sigma_{3}\right)$ select the parts of the multivector $\psi$ that are parallel (+) or antiparallel (-) to k. Thus, we have two good quantum numbers, the wave vector $k>0$ and helicity $h= \pm 1$.

From the projective representation, Eq. (2), follows that $\psi$ can be written a sum of two parts having opposite helicities: $\psi=\psi_{+}+\psi_{-}$. Then, the respective eigenenergies $E_{+}$and $E_{-}$can be found from two independent multivector eigenequations, $H_{30}\left(\psi_{ \pm}\right)=E_{ \pm} \psi_{ \pm}$, or in a full form

$$
\frac{k h_{ \pm}}{2}\left(\psi_{ \pm} \pm \hat{\mathbf{k}} \psi_{ \pm} \sigma_{3}\right)=E_{ \pm} \psi_{ \pm} .
$$

This equation can be rewritten in a form of rotor equation, $R \hat{\mathbf{a}} \widetilde{R}=\hat{\mathbf{b}}$, where $\hat{\mathbf{a}}$ and $\hat{\mathbf{b}}$ are two unit vectors in $3 \mathrm{D}$ space and $R$ is the rotor which satisfies $R \widetilde{R}=\widetilde{R} R=1$. The tilde denotes the reversion operation [1]. Thus, Eq. (3) in rotor form is 


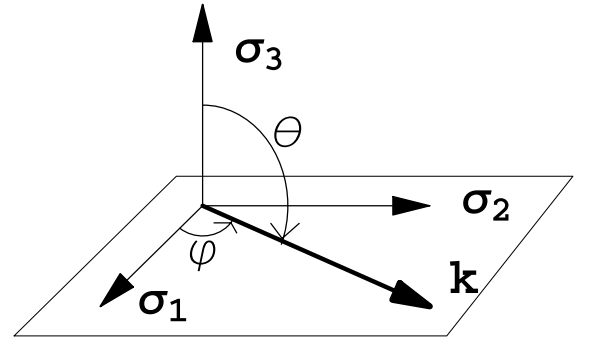

Fig. 1. $\mathrm{Cl}_{3,0}$ basis vectors $\boldsymbol{\sigma}_{k}$ and wave vector $\mathbf{k}$ with respect to graphene plane. Rotation of $\sigma_{3}$ towards $\pm \mathbf{k}$ gives the eigenspinors of the problem.

$$
\psi_{ \pm} \sigma_{3} \widetilde{\psi}_{ \pm}= \pm \hat{\mathbf{k}}
$$

where $\psi$ should be normalized $\tilde{\psi} \psi=\psi \tilde{\psi}=1$. The property that $\psi$ represents the rotor allows one to express $\psi_{+}$as a rotation of $\sigma_{3}$ in the bivector plane that is made up of vectors $\hat{\mathbf{k}}$ and $\boldsymbol{\sigma}_{3}$, Fig. 1 ,

$$
\psi_{ \pm}=\exp \left( \pm \hat{\mathbf{k}} \wedge \sigma_{3} \pi / 4\right)=\frac{1}{\sqrt{2}}\left(1 \pm \hat{\mathbf{k}} \wedge \sigma_{3}\right)
$$

where the wedge (or outer) product $\hat{\mathbf{k}} \wedge \sigma_{3}=\left(\hat{\mathbf{k}} \sigma_{3}-\right.$ $\left.\boldsymbol{\sigma}_{3} \hat{\mathbf{k}}\right) / 2$ represents the bivector. If the obtained spinors are inserted back into Hamiltonian (2), the conduction and valence band eigenenergies are satisfied: $\widetilde{\psi}_{ \pm} H_{30}\left(\psi_{ \pm}\right)=h_{ \pm} k= \pm k$.

Since the helicity is a good quantum number, one can make a superposition of two states having opposite helicities:

$$
\psi_{\beta}=\cos \beta \psi_{+}+\sin \beta \psi_{-}, \quad \tilde{\psi}_{\beta} \psi_{\beta}=1,
$$

where $\beta$ is a mixing, angle. Then the energy of this state is found to be

$$
\widetilde{\psi}_{\beta} H_{30}\left(\psi_{\beta}\right)=k \cos 2 \beta .
$$

This formula shows that the electron energy can be varied in two ways, either by changing the wave vector magnitude, or by making an appropriate superposition with opposite helicities.

\section{Graphene in $\mathrm{Cl}_{3,1}$ algebra with spin included}

$\mathrm{Cl}_{3,1}$ consists of 16 basis elements: scalar, a set of four vectors $\left\{\mathbf{e}_{1}, \mathbf{e}_{2}, \mathbf{e}_{3}, \mathbf{e}_{4}\right\}$, two sets of bivectors $\left\{\mathbf{e}_{12}, \mathbf{e}_{23}, \mathbf{e}_{31}\right\}$ and $\left\{\mathbf{e}_{14}, \mathbf{e}_{24}, \mathbf{e}_{34}\right\}$, four trivectors $\left\{I \mathbf{e}_{1}, I \mathbf{e}_{2}, I \mathbf{e}_{3}, I \mathbf{e}_{34}\right\}$, and finally of pseudoscalar $I \equiv \mathbf{e}_{1234}$, where abbreviation $\mathbf{e}_{1} \mathbf{e}_{2} \equiv \mathbf{e}_{12}$ etc. was used. The signature is determined by squares of vectors: $\mathbf{e}_{i}^{2}=1$ for $i=1,2,3$ and $\mathbf{e}_{4}^{2}=-1$.

With electron spin included, the Hamiltonian matrix of a monolayer graphene is usually described in the basis $\left\{A_{\uparrow}, A_{\downarrow}, B_{\uparrow}, B_{\downarrow}\right\}$, where $A$ and $B$ are inequivalent carbon atoms in an elementary cell, and up and down arrows indicate electron spin states. The equivalent Hamiltonian function in GA that describes such Hamiltonian is [5]

$$
H_{31}(\psi)=\left(\eta k_{x} \mathbf{e}_{1}+k_{y} \mathbf{e}_{2}\right) \psi I \mathbf{e}_{3},
$$

where $\eta=1$ for $K$-valley, and $\eta=-1$ for $K^{\prime}$-valley. $\psi$ is the spinor which consists of sum of even grade elements (scalar + pseudoscalar + bivectors). In the following we shall take into account two interaction mechanisms related with spin, namely, spin-orbit (SO) Rashba and exchange interactions characterized by parameters $\lambda_{\mathrm{R}}$ and $M$. The respective interaction Hamiltonian in GA reads [5]:

$$
H_{i}(\psi)=\frac{\lambda_{R}}{2}\left(-\mathbf{e}_{1} \psi \mathbf{e}_{3}+\eta \mathbf{e}_{14} \psi \mathbf{e}_{34}\right)+M \mathbf{e}_{3} \psi \mathbf{e}_{3}
$$

where $\lambda_{\mathrm{R}}$ and $M$ are measured in units of the nearest neighbour hopping parameter. The time-dependence of $\psi$ is described by

$$
\partial_{t} \psi \mathbf{e}_{12}=H_{31}(\psi)+H_{i}(\psi) \equiv H(\psi)
$$

It should be noted that Eq. (10) is not of Diractype. In the relativistic equation instead of the bivector $\mathbf{e}_{12}$ there appears the spacelike vector $\mathbf{e}_{4}$ the square of which gives -1 [1] . Figure 2 shows the spectrum of considered Hamiltonian $H(\psi)$.

To solve (10) and respective eigen-multivector equation $H\left(\psi_{i}\right)=E_{i} \psi_{i}$, where $i$ is the band index, we introduce the spatial inversion operation denoted by a bar over the multivector $M$,

$$
\bar{M}=-\mathbf{e}_{4} M \mathbf{e}_{4}
$$

It satisfies the following properties: $\overline{\mathbf{e}}_{i}=-\mathbf{e}_{i}$ if $i=1,2,3$; $\overline{\mathbf{e}}_{4}=\mathbf{e}_{4} ; \bar{M}_{i} M_{j}=\bar{M}_{i} \bar{M}_{j}$. The spatial inversion allows to divide the general bispinor into two parts: $\psi=\psi_{+}+\psi_{-}$,

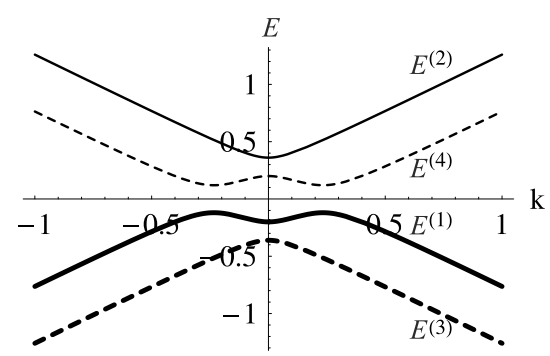

Fig. 2. Spectrum of monolayer graphene with Rashba and exchange interactions included $\left(\lambda_{\mathrm{R}}=0.3 \mathrm{M}=0.2\right)$. The wave vector axis is normalized to $k_{\mathrm{c}}=2 \pi / a$ ( $a$ is the graphene lattice constant). 
where

$$
\begin{aligned}
& \psi_{+}=a_{0}+a_{1} \mathbf{e}_{23}+a_{2} \mathbf{e}_{13}+a_{3} \mathbf{e}_{12}, \\
& \psi_{-}=I\left(-b_{0}+b_{1} \mathbf{e}_{23}+b_{2} \mathbf{e}_{13}+b_{3} \mathbf{e}_{12}\right),
\end{aligned}
$$

and where $a_{i}$ and $b_{i}$ are real. If applied to $\psi$, the spatial inversion gives $\bar{\psi}=\psi_{+}-\psi_{-}$. The division of even and odd parts allows to introduce respective rotors in the subspace spanned by spatial basis vectors $\mathbf{e}_{1}, \mathbf{e}_{2}$, and $\mathbf{e}_{3}$, i. e. although full $\psi$ does not represent the rotor in $\mathrm{Cl}_{3,1}$, nonetheless, its parts $\psi_{+}$and $I \psi_{-}$do. Their normalization is $\widetilde{\psi}_{+} \psi_{+}=a_{0}^{2}+a_{1}^{2}+a_{2}^{2}+a_{3}^{2}$ and $\left(\widetilde{I}_{-}\right)\left(I \psi_{-}\right)=$ $b_{0}^{2}+b_{1}^{2}+b_{2}^{2}+b_{3}^{2}$. As we shall see, the knowledge of rotors, as in $\mathrm{Cl}_{3,0}$ algebra, allows one to construct the eigenmultivectors.

Using the eigenequation $H(\psi)=E \psi$ and its inverse $\bar{H}(\psi)=E \bar{\psi}$ one can construct, for example, the following rotor equation for even spinor $\psi_{+}$,

$$
\frac{\psi_{+}^{(i)} \tilde{e}_{3}^{(i)}}{\left|\psi_{+}\right|^{2}}=\mathrm{a}^{(i)}
$$

as shown in Fig. 3, where the vector $\mathbf{a}^{(i)}$ is related with the $i$-th band eigenenergy $E^{(i)}$,

$$
\begin{aligned}
& \mathrm{a}^{(i)}\left[\left(E^{(i)}\right)^{2} k^{2}\left(4 M^{2}+\lambda_{\mathrm{R}}^{2}\right)\right]= \\
& E^{(i)} \lambda_{\mathrm{R}}\left[\left(E^{(i)}\right)^{2}-k^{2}-M^{2}\right]\left(\mathbf{k e}_{1} \mathrm{k}\right)- \\
& E^{(i)} M\left[2\left(E^{2}-k^{2}-M^{2}\right)+\lambda_{\mathrm{R}}^{2}\right]\left(\mathbf{k e}_{3} \mathrm{k}\right) .
\end{aligned}
$$

Now $\psi_{+}^{(i)} /\left|\psi_{+}^{(i)}\right|=R_{+}^{(i)}$ are rotors that satisfy $R_{+}^{(i)} \widetilde{R}_{+}^{(i)}=1$.

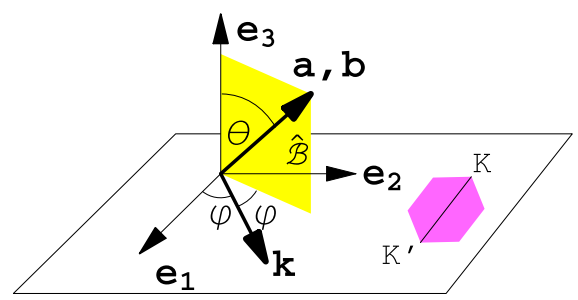

Fig. 3. Basis vectors $\mathbf{e}_{1}, \mathbf{e}_{2}$, and $\mathbf{e}_{3}$, wave wector $\mathbf{k}$, and plane $\hat{\boldsymbol{B}}$ that contains vectors $\mathbf{e}_{3}$ and $\mathbf{a}$ (or $\left.\mathbf{b}\right)$. The line that connects $K$ and $K^{\prime}$ valleys in the first Brillouin zone is parallel to $\mathbf{e}_{1}$.

The vectors $\mathbf{e}_{3}$ and $\mathbf{a}^{(i)}$ allow to construct the unit rotation plane (bivector $\hat{\boldsymbol{B}}^{(\mathrm{i})}$ in Fig. 3) and rotation angle $\theta$ using the standard GA rules [5]. Similar calculations give the second rotor $\left(I \psi_{-}^{(\mathrm{i})}\right)$. The total bispinor of the $\mathrm{i}$-th band then is equal to sum $\psi^{(i)}=\psi_{+}^{(i)}+\psi_{-}^{(i)}$. Below a few examples- are presented how bispinor $\psi^{(i)}$ can be applied to calculate electron spin and Berry phase.

Spin. The average spin components in $\mathrm{Cl}_{3,1}$ are

$$
\begin{aligned}
& S_{1}^{(i)}=-\left\langle\bar{\psi}^{(i)} \mathbf{e}_{34} \psi^{(i)}\right\rangle, \\
& S_{2}^{(i)}=-\left\langle\bar{\psi}^{(i)} I \mathbf{e}_{4} \psi^{(i)} \mathbf{e}_{4}\right\rangle, \\
& S_{3}^{(i)}=-\left\langle\bar{\psi}^{(i)} I \mathbf{e}_{3} \psi^{(i)} \mathbf{e}_{3}\right\rangle,
\end{aligned}
$$

where the brackets indicate the scalar part. The true values should be multiplied by $\hbar / 2$. Figure 4 illustrates a spin field calculated with (15) and band parameters $M=0.05, \lambda_{\mathrm{R}}=0.2$.

If $\mathrm{SO}$ interaction is switched off, $\lambda_{\mathrm{R}}=0$, the a spin field reduces to a very simple form $\mathbf{S}_{K}^{(i)}=\left(S_{1}^{(i)}, S_{2}^{(i)}, S_{3}^{(i)}\right)=$ $(0,0, \pm 1)$ where the plus sign is for $i=1,4$ and the minus sign for $i=2,3$ bands, Fig. 2. Thus, now the lowest conduction $E^{(4)}$ and the highest valence $E^{(1)}$ bands have the same spin direction perpendicular to graphene plane.

When $M=0$, the spin formulas simplify to

$$
\mathbf{S}_{K}^{(i)}=\frac{2 E^{(i)} k}{E^{(i) 2}+k^{2}}(\sin \varphi,-\cos \varphi, 0),
$$

i. e. in this case the average spin lies in the graphene plane and simultaneously is perpendicular to $\mathbf{k}=k(\cos \varphi, \sin \varphi, 0)$. At large $\mathbf{k}$ values, when $E^{(\mathrm{i})} \sim k$, the spin magnitude approaches free-space value.

It can be shown that the scalar product of $\mathbf{S}_{K}^{(i)}$ and $\mathbf{k}$ is always equal to zero, if $\lambda \neq 0$ and $M \neq 0$. Therefore, the spin will always remain perpendicular to the wave vector. The additional degree of freedom which determines whether spin lies in or is perpendicular to graphene plane (or takes an intermediate position) depends on dispersion of a particular band.

Berry phase. In $\mathrm{Cl}_{3,1}$ the Berry phase is [5]

$$
\Gamma=-\mathbf{e}_{12} \lim _{N \rightarrow \infty} \sum_{j=0}^{N-1} \log \left\langle\psi_{j} \psi_{j+1}\right\rangle .
$$

We shall assume that points $j$ lie on the circle around the energy valley and thus can be described by a single parameter, the azimuthal angle $\phi_{j}$. The matrix element $\left\langle\bar{\psi}_{j} \psi_{j+1}\right\rangle$ between adjacent points can be calculated by expanding the bispinor $\psi_{j}$ in the vicinity of $\phi_{j}$. Then to the first order in $\delta \phi$ we find that $\left\langle\bar{\psi}_{j} \psi_{j+1}\right\rangle \approx 1$ and $\left\langle\overline{\psi_{j}} \psi_{j+1} \mathbf{e}_{12}\right\rangle \approx-\beta^{(i)} \delta \phi$, so that

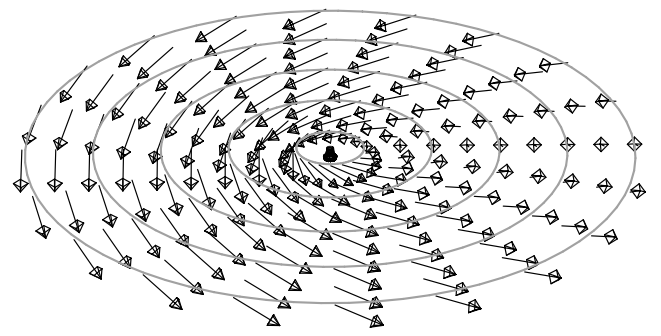

Fig. 4. Field of spins represented in $k_{x}-k_{y}$ plane for upper $E^{(1)}$ valence band. 


$$
\left\langle\bar{\psi}_{j} \psi_{j+1}\right\rangle=-\beta^{(i)} \delta \phi \mathbf{e}_{12}
$$

where $\beta^{(i)}$ is a function of $k, \lambda_{\mathrm{R}}$ and, $M$. Calculations show that $\beta^{(1)}=\beta^{(4)}$ and $\beta^{(2)}=\beta^{(3)}$, i. e. we have the same Berry phase for conduction and valence bands having similar dispersion character. Insertion of (18) into (17) gives the Berry phase

$$
\Gamma^{(i)}=-\mathbf{e}_{12} \beta^{(i)} \sum_{j=0}^{N-1} \delta \phi \mathbf{e}_{12}=2 \pi \beta^{(i)} .
$$

We find that $\Gamma^{(1)}=\Gamma^{(4)}$ and $\Gamma^{(2)}=\Gamma^{(3)}$. When one of interaction mechanisms is turned off, the expression for the Berry phase reduces to a simple form: $\Gamma=3 \pi$ if $\lambda_{\mathrm{R}}=0$, and $\Gamma=2 \pi$ if $M=0$, which are independent of the band index. Figure 5 shows the dependence of $\Gamma^{(i)}$ on $k$. The steps in $\Gamma^{(i)}$ are related with energy minima/

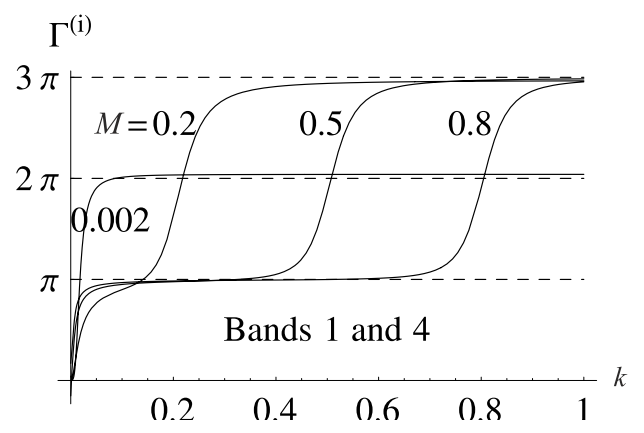

Fig. 5. Berry phase vs. wave vector in valence $E^{(1)}$ and conduct ion $E^{(4)}$ bands of Fig. 2. $\lambda_{\mathrm{R}}=0.1 ; M=0.002,0.2$, $0.4,0.6$. maxima in the respective spectra, Fig. 2. Calculations show that Berry phases for $K$ - and $K^{\prime}$-valleys are the same.

In conclusion, the relativistic Minkowski's spacetime algebra $\mathrm{Cl}_{3,1}$ and its subalgebra $\mathrm{Cl}_{3}$ can be used to analyse graphene properties. Earlier [4] it has been shown that the smaller $\mathrm{Cl}_{2,0}$ algebra which describes the true flatland is unsuitable for this purpose.

\section{Acknowledgements}

This work in part was supported by Agency for Science, Innovation and Technology (grant No 31V-32) in the framework of High Technology Development Programme for 2011-2013.

\section{References}

[1] C. Doran and A. Lasenby, Geometric Algebra for Physicists (Cambridge University Press, Cambridge, 2003)

[2] A. Dargys, Optical Mueller matrices in terms of geometric algebra, Opt. Commun. 285, 4785-4792 (2012).

[3] A. Dargys, Quantum ring in the eyes of geometric (Clifford) algebra, Physica E 47, 47-50 (2013).

[4] A. Dargys, Quantum flatland and monolayer grap. from a viewpont of geometric algebra, Acta Phys. Pol. A 124(4) 732-739 (2013).

[5] A. Dargys, Spin and pseudospin in monolayer graphene, Phys. Scr. (2014) accepted.

\title{
CLIFFORDO ALGEBROS TAIKYMAS GRAFENO ANALIZEI
}

\author{
A. Dargys \\ Fiziniu ir technologijos mokslu centro Puslaidininkiu fizikos institutas, Vilnius, Lietuva
}

\section{Santrauka}

Remiantis Cliffordo algebra išnagrinètos vienasluoksnio grafeno savybės. Parodyta, kad mažiausia iš Cliffordo algebrų, su kuria dar galima analizuoti grafeną, yra $\mathrm{Cl}_{3,0}$ algebra, nusakanti trimatę Euklido erdvę. Kaip parodyta anksčiau [4], plokštumos algebra $\mathrm{Cl}_{2,0}$ yra per maža, kad galètų aprašyti kvantines grafeno savybes. Jei papildomai reikia îskaityti elektrono sukini, minètos
$\mathrm{Cl}_{3,0}$ algebros jau nepakanka. Darbe parodyta, kad tokiu atveju patogu naudotis didesne $\mathrm{Cl}_{3}$ algebra, kuri aprašo reliatyvistinę Minkowskio erdvę. $\mathrm{Cl}_{3,0}$ ir $\mathrm{Cl}_{3,1}$ algebrų atvejais nustatyta, kad elektrono banginę funkciją galima sukonstruoti su kiekvienos iš algebrų rotoriais. Gautos atitinkamos rotorių išraiškos. Pasitelkus $\mathrm{Cl}_{3,1}$ algebrą, išnagrinètos elektrono sukinio ir Berry'io fazès savybės. 